\title{
Thermodynamics of $N_{f}=2$ QCD on anisotropic lattices
}

Yusuke Namekawa*†

Department of Physics, Nagoya University, Nagoya 464-8602, Japan

E-mail: namekawa@het.ph.tsukuba.ac.jp

\section{Y. Kikukawa}

Institute of Physics, University of Tokyo, Komaba, Tokyo 153-8902, Japan

\begin{abstract}
The phase structure of $N_{f}=2 \mathrm{QCD}$ is studied by lattice simulations. We employ the RG improved gauge and clover fermion action on $8^{3} \times 8,10^{3} \times 10$ and $12^{3} \times 12$ lattices with the anisotropy $\xi \equiv a_{s} / a_{t}=2$, where $a_{s}$ and $a_{t}$ are spatial and temporal lattice spacings. We determine the critical temperature $T_{c}$ by the Polyakov loop susceptibility. Hadron properties at finite temperature are also investigated. Our lattice wavefunctions imply pseudoscalar and vector mesons are bound states even around the phase transition point. We found meson masses near the transition point do not show significant shifts by thermal effects.
\end{abstract}

The XXV International Symposium on Lattice Field Theory

July 30 - August 42007

Regensburg, Germany

\footnotetext{
* Speaker.

${ }^{\dagger}$ Present address : Center for Computational Sciences, University of Tsukuba, Tsukuba, Ibaraki 305-8577, Japan
} 


\section{Introduction}

Numerical simulations based on the lattice QCD are powerful tools to investigate the strongly coupled quark gluon plasma. Lattice QCD can give non-perturbative results in a model independent way. The phase structure of QCD as well as thermodynamic properties have been studied [1]. Most finite temperature QCD simulations have been performed with the staggered fermion. Numerical costs for the staggered fermion are relatively small, compared with other fermion formulations. $N_{f}=2+1$ QCD simulations including dynamical up, down and strange quarks have been carried out by many collaborations. They showed that $N_{f}=2+1 \mathrm{QCD}$ has a crossover at the physical point [2] and the crossover temperature in the continuum limit is $150-190[\mathrm{MeV}]$ [1]. A subtle point of the staggered fermion is the fourth-root trick of the quark determinant, which makes the action non-local and complicates the continuum extrapolation [3]. Cross checks with different fermion formulations are desirable.

In contrast to the case of the staggered fermions, the Wilson fermion has a simple structure on quark flavors. The Wilson fermion does not need the fourth-root trick. Pseudo-order parameters satisfy the $O(4)$ scaling well with the RG improved gauge action [4], which leads to a reliable chiral extrapolation. But, simulations using the Wilson fermions are limited due to their computational demands. Only one group reported the chiral and continuum limit value of critical temperature $T_{c}=166-173[\mathrm{MeV}][5]$.

In this work, we study the phase structure of $N_{f}=2$ QCD using the clover improved Wilson fermion combined with the RG improved gauge action. We employ anisotropic lattices with smaller temporal lattice spacing than the spatial one to reduce discretization effects of thermodynamic quantities and to obtain more data points in the temporal direction. Simulations are performed on $8^{3} \times 8,10^{3} \times 10$ and $12^{3} \times 12$ lattices. We determine pseudo-critical points by the Polyakov loop susceptibility. The critical temperature in the chiral limit is obtained by chiral extrapolations of pseudo-critical points using $O(4)$ scaling function. We also check the existence of bound states of mesons by measuring their wavefunctions, and obtain pseudoscalar and vector meson masses around the phase transition point.

\section{Method}

We performed full QCD simulations with two flavors of degenerate up and down quarks. For the gauge part, we employ the RG improved action defined by

$$
S_{g}=\beta\left\{\frac{1}{\gamma_{G}} \sum_{x, i, j} c_{0} P_{i j}(x)+c_{1}\left(R_{i j}(x)+R_{j i}(x)\right)+\gamma_{G} \sum_{x, i} c_{0} P_{i 4}(x)+c_{1}\left(R_{i 4}(x)+R_{4 i}(x)\right)\right\},
$$

where $i, j$ runs in spatial directions and $P_{\mu v}$ and $R_{m u v}$ are the plaquette and rectangular loop in the $\mu-v$ plane. $c_{0}=3.648$ and $c_{1}=-0.331$ are determined by a renormalization group analysis [6]. They satisfy the normalization condition $c_{0}+8 c_{1}=1$, and $\beta=6 / g^{2} \cdot \gamma_{G}$ is the bare anisotropy, which is tuned non-perturbatively so that the ratio of spatial and temporal lattice spacings becomes two, $\xi \equiv a_{s} / a_{t}=2$ [7]. For the fermion part, we use the clover quark action defined by

$$
S_{q}=\sum_{x, y} \bar{q}_{x} D_{x, y} q_{y}
$$


Table 1: Simulation parameters.

\begin{tabular}{cccc}
\hline size & $\beta$ & $\kappa$ & traj \\
\hline $8^{3} \times 8$ & $1.95-2.1$ & $0.1100-0.1275$ & $1000-7000$ \\
$12^{3} \times 8$ & 2.0 & $0.1170-0.1265$ & $1000-7000$ \\
\hline $10^{3} \times 10$ & $2.0-2.1$ & $0.1170-0.1270$ & $1000-4000$ \\
\hline $12^{3} \times 12$ & $2.05-2.1$ & $0.1170-0.1275$ & $500-6000$ \\
\hline
\end{tabular}

$$
\begin{aligned}
D_{x, y} & =\delta_{x y}-\kappa_{s} \sum_{i}\left[\left(r-\gamma_{i}\right) U_{x, i} \delta_{x+\hat{i}, y}+\left(r+\gamma_{i}\right) U_{x, i}^{\dagger} \delta_{x, y+\hat{i}}\right] \\
& -\kappa_{t}\left[\left(1-\gamma_{i}\right) U_{x, 4} \delta_{x+\hat{4}, y}+\left(1+\gamma_{i}\right) U_{x, 4}^{\dagger} \delta_{x, y+\hat{4}}\right]-\kappa_{s}\left[r c_{s} \sum_{i, j} F_{i j}(x) \sigma_{i j}+c_{t} \sum_{i} F_{i 4}(x) \sigma_{i 4}\right]
\end{aligned}
$$

where $\kappa_{s}$ is the hopping parameter in the spatial direction and $\kappa_{t}$ is the one in the temporal direction. $F_{\mu \nu}$ is the clover-shaped lattice discretization of the field strength and $\sigma_{\mu v} \equiv(i / 2)\left[\gamma_{\mu}, \gamma_{v}\right]$. For the clover coefficients, we adopt a meanfield improved value $c_{s}=1 / u_{s}^{3}, c_{t}=1 / u_{s} u_{t}^{2}$ with $u_{s}=(1-1.154 / \beta)^{1 / 4}, u_{t}=1$. We set the spatial Wilson parameter $r=1 / \xi$. As in the gauge sector, the bare anisotropy $\gamma_{F} \equiv \frac{\kappa_{t} u_{t}}{\kappa_{s} u_{s}}$ is tuned to satisfy $\xi=2$ using the dispersion relation [7]. For convenience, we define $\kappa$.

$$
\frac{1}{\kappa} \equiv \frac{1}{\kappa_{s} u_{s}}-2\left(\gamma_{F}+3 r-4\right)
$$

Numerical simulations are performed at $\beta=1.9-2.1$ on $8^{3} \times 8,10^{3} \times 10$ and $12^{3} \times 12$ lattices using the Hybrid Monte Carlo algorithm. The hopping parameters are chosen as $\kappa=0.1100$ 0.12750 , which cover the pi-rho ratios $m_{P S} / m_{V}=0.75-0.60$ at zero temperature. The Polyakov loops are measured at each trajectory and hadron masses are measured at each 5 trajectories. Statistical errors are estimated by the jackknife method with bins of 100 trajectories. Our simulation parameters are summarized in Table 1.

\section{Critical temperature}

We determine the pseudo-critical temperature from the peak location of the Polyakov loop susceptibility, which is an order parameter in the heavy quark mass limit. Two-flavor QCD is expected to have the second order phase transition at the chiral limit of zero quark masses. However, the phase transition is smeared out as quark masses increase, and it becomes crossover. Since crossover does not have any order parameters, we identify the pseudo-phase transition points where fluctuations grow. The Polyakov loop $L$ is built of temporal link variables.

$$
L \equiv \operatorname{Tr} \prod_{t} U_{4}(\mathbf{x}, t) .
$$

We perform $\mathrm{Z}(3)$ rotation to limit the phase of $L$ to a range $[-\pi / 3,+\pi / 3]$. The Polyakov loop susceptibility $\chi_{L}$ in a spatial volume $V$ is defined as $\chi_{L} \equiv V\left(\left\langle L^{2}\right\rangle-\langle L\rangle^{2}\right)$. Fig. 1 illustrates typical $\kappa$ dependence of the Polyakov loop values and susceptibilities. The peak location of $\chi_{L}$ is obtained 

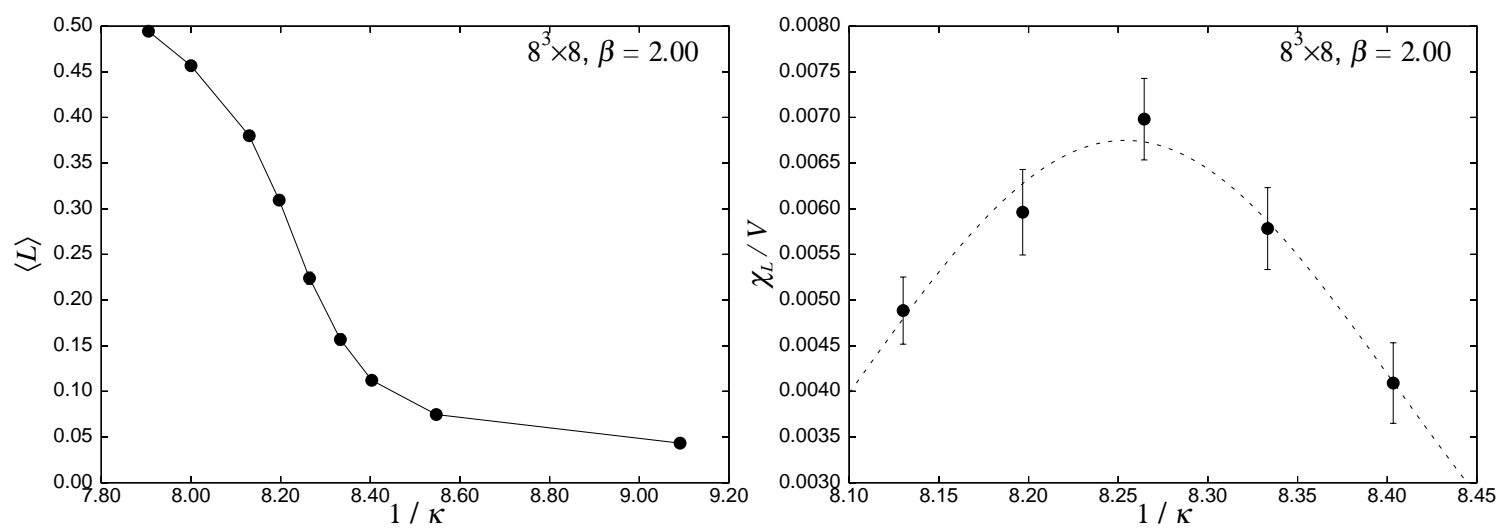

Figure 1: $\kappa$ dependence of the Polyakov loop (left panel) and its susceptibility (right panel) at $\beta=2.00$ on $8^{3} \times 8$ lattice. The peak location of $\chi_{L}$ is obtained by Gaussian fits to the data around it.

by Gaussian fits to the data around it, which we consider as a pseudo-phase transition point on the lattice.

We checked the finite size effects for the pseudo-critical temperature. In a finite size box, singularities associated with phase transitions become mild. It is necessary to check magnitude of finite size effects. We performed runs on $8^{3} \times 8$ and $12^{3} \times 8$ lattices and found that the peak locations of $\chi_{L}$ agree within $2 \sigma$. We don't find any clear sign of finite size dependence. It is consistent that $N_{f}=2$ finite temperature QCD has crossover at our simulation point. But, more systematic study of finite size scaling is needed to confirm it.

We employ $O(4)$ scaling function for chiral extrapolations of pseudo-phase transition points. $O(4)$ scaling is expected from the universality at the chiral limit [8]. $O(4)$ scaling function is

$$
\beta_{p c}=\beta_{c t}+B\left(m_{q}^{V W I}\right)^{1 / \beta \delta}
$$

where the critical exponent is obtained by $O(4)$ spin model, $1 / \beta \delta=0.537(7)$ [9]. We adopt the vector Ward identity quark mass $m_{q}^{V W I}=\left(1 / \kappa_{p c}-1 / \kappa_{c}\right) / 2$ as an external field. $\beta_{p c}$ and $\kappa_{p c}$ are $\beta$ and $\kappa$ at the pseudo-phase transition point. $\beta_{c t}$ and $B$ are free parameters. We fit our lattice data to this $O(4)$ scaling function. As in the case on isotropic lattices, $O(4)$ scaling is satisfied well for data on anisotropic lattices with the RG gauge and clover fermion action combination. The situation is illustrated in Fig. 2. Once $\beta_{c t}$ is obtained from the chiral extrapolation, we can convert it to the critical temperature $T_{c}$.

$$
T_{c}=\frac{1}{N_{t} a_{t}\left(\beta_{c t}\right)},
$$

where the temporal lattice scale $a_{t}(\beta)$ is set by the rho meson mass at the zero temperature. Fig. 2 plots temporal size dependence of $T_{c}$. Our preliminary data predicts $T_{c} \sim 160 \mathrm{MeV}$ in the continuum limit. Our value for $T_{c}$ is slightly smaller than those estimated on isotropic lattices. Anisotropic lattices may remove discretization errors of $T_{c}$ in a more efficient way than isotropic lattices.

\section{Spectroscopy}

We examined temperature dependences of light hadron masses. After we checked that hadrons keep to be bound states, light hadron spectroscopies are performed around the pseudo-critical tem- 

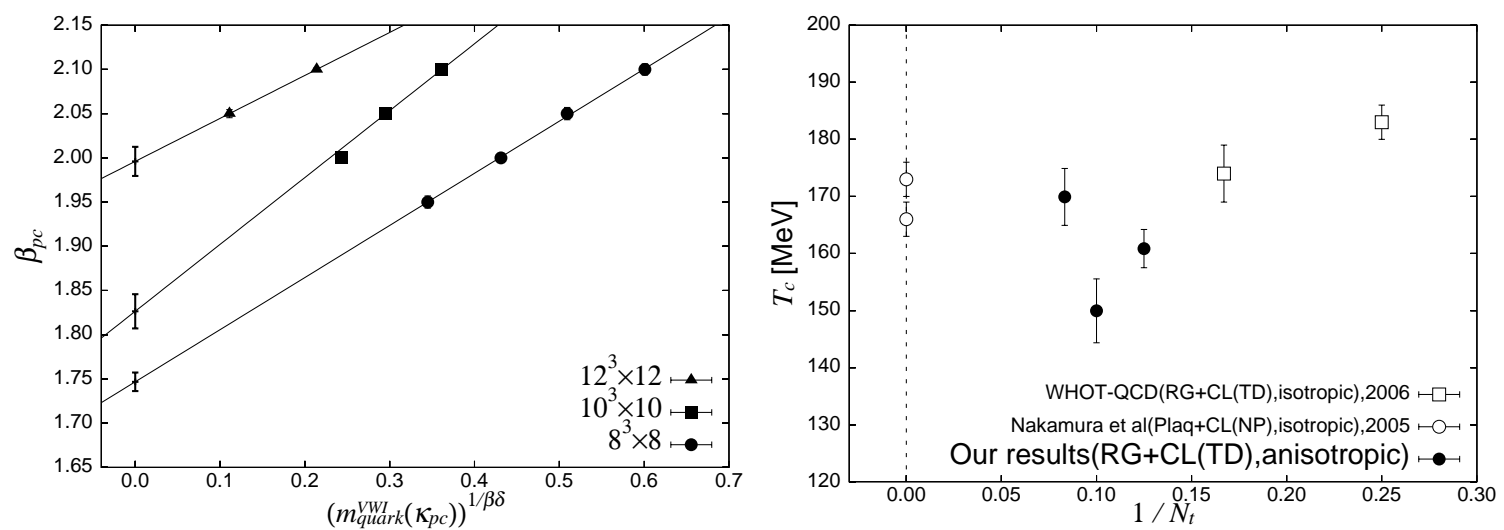

Figure 2: Chiral extrapolation of the pseudo-critical points (left panel) and continuum extrapolation of the critical temperature (right panel). For comparison, critical temperatures on isotropic lattices also plotted [4, 5].

perature and zero temperature. On conventional isotropic lattices, it is hard to extract masses from temporal correlators, because the temporal length is limited by the temperature. Anisotropic lattices have an advantage in calculating masses, due to finer resolution in the temporal direction.

First, we observed a meson wavefunction around the pseudo-critical point to investigate hadrons are bound states or not. If hadrons are bound states, their wavefunctions must be spatially localized. If not, the wavefunctions are wide spread in space. A meson wavefunction $\phi(\mathbf{r})$ is constructed of a quark field $q(\mathbf{x}, t)$ as follow.

$$
\phi(\mathbf{r})=\frac{\sum_{\mathbf{x}}\left\langle\bar{q}(\mathbf{x}, t) \Gamma q(\mathbf{x}+\mathbf{r}, t)(\bar{q}(\mathbf{x}, t) \Gamma q(\mathbf{x}, t))^{\dagger}\right\rangle}{\sum_{\mathbf{x}}\left\langle\bar{q}(\mathbf{x}, t) \Gamma q(\mathbf{x}, t)(\bar{q}(\mathbf{x}, t) \Gamma q(\mathbf{x}, t))^{\dagger}\right\rangle}, \quad \Gamma=\gamma_{5}, \gamma_{i}, i=1,2,3 .
$$

where $t=N_{t} / 2$ is employed and three polarizations are averaged for a vector meson. Wavefunctions of pseudoscalar and vector mesons at zero and finite temperatures are shown in Fig. 3. As in the case of zero temperature, the wavefunctions are exponentially localized in space. Our results imply that mesons are bounded even near the pseudo-phase transition point.

Then, we calculated meson spectrum. Since we are interested in the temperature dependence of the ground state energy, we enhance the ground state signal using the wavefunction. Meson operators $M(\mathbf{x}, t)$ are smeared by wavefunctions.

$$
M(\mathbf{x}, t)=\sum_{\mathbf{r}, \mathbf{r}^{\prime}} \phi_{\Gamma}(\mathbf{r}) \phi_{\Gamma}\left(\mathbf{r}^{\prime}\right) \bar{q}(\mathbf{x}+\mathbf{r}, t) \Gamma q\left(\mathbf{x}+\mathbf{r}^{\prime}, t\right) .
$$

Point operator is realized by setting $\phi_{\Gamma}(\mathbf{r})=\delta_{\mathbf{r}, \mathbf{0}}$. We can extract meson masses from correlation functions. $G(t)=\sum_{\mathbf{x}}\left\langle M(\mathbf{x}, t) M^{\dagger}(\mathbf{0}, 0)\right\rangle$. Fig. 4 is an example of effective masses, $m^{\text {eff }} \equiv$ $\log \frac{G(t)}{G(t+1)}$. Smearing meson operators allow us to extract their masses from small $t$ region. Correlation functions in the plateau of their effective masses are fitted with cosh to obtain masses.

After computing meson masses at finite temperature, we compare them with those at zero temperature. The ratios of finite and zero temperature masses are plotted in Fig. 5. Out data of vector meson masses on $N_{t}=8$ does not show any clear dependence toward zero mass. It contradicts the 

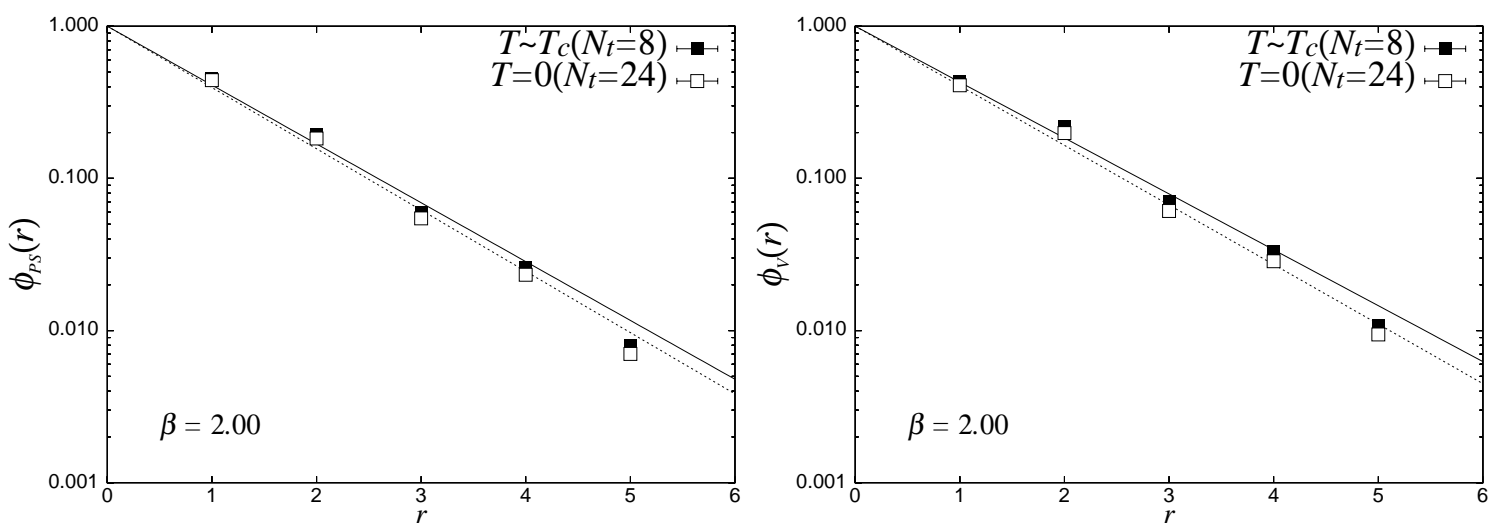

Figure 3: Pseudoscalar(left panel) and vector meson wavefunctions(right panel) at zero and finite temperatures.
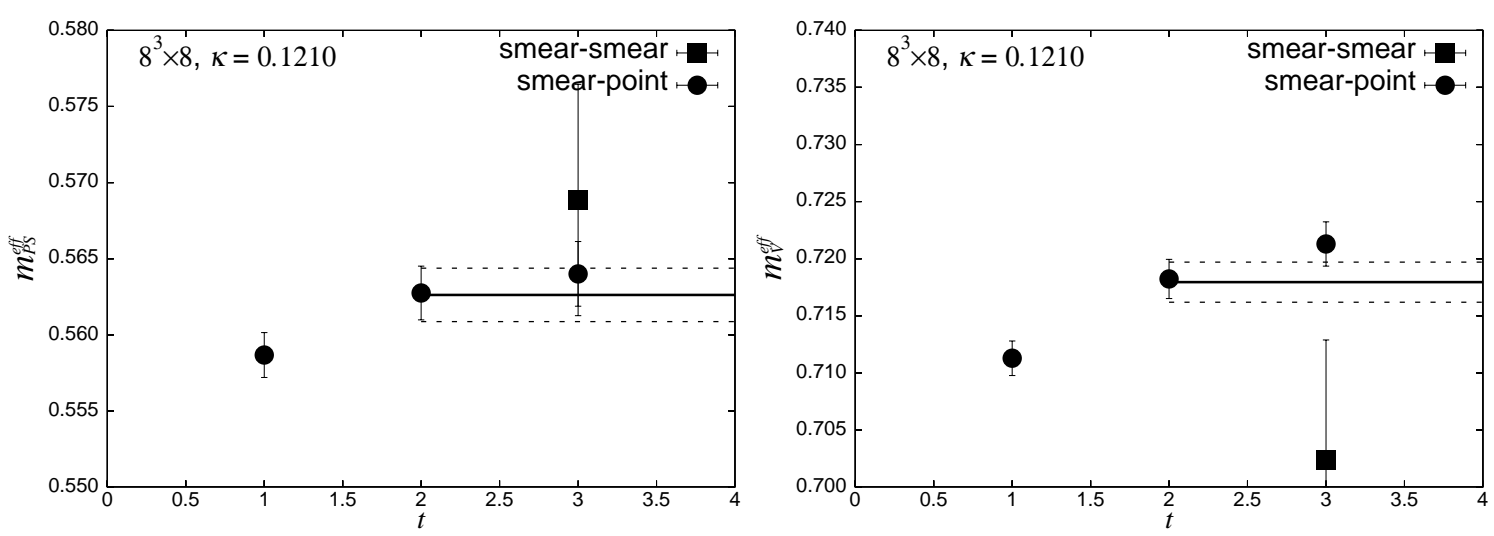

Figure 4: Pseudoscalar(left panel) and vector meson effective masses(right panel) at finite temperatures.

prediction of the vector manifestation scenario that vector meson masses approach to zero in the chiral limit of zero quark masses at the critical temperature [10]. One of possibilities that conceals the decrease of vector meson masses toward the chiral limit is a lattice artifact associated with a finite lattice spacing. $O(a)$ may modify the chiral behavior. To clarify this point, calculations at finer lattice spacings are needed. Computations on $N_{t}=10$ and 12 lattices are ongoing.

\section{Conclusions}

We investigated the phase structure of $N_{f}=2$ QCD using the RG-improved gauge action and the clover-improved Wilson quark action on $\xi=2$ anisotropic lattices. We determined pseudocritical points at finite quark masses by the Polyakov loop susceptibilities. The pseudo-critical points are found to be consistent with $O(4)$ scaling. The critical temperature in the chiral limit is obtained using $O(4)$ scaling function. Our value of the critical temperature is $T_{c} \sim 160 \mathrm{MeV}$, which is slightly lower than those obtained on isotropic lattices. Anisotropic lattices may reduce discretization errors of $T_{c}$ efficiently. For more precise determination of $T_{c}$, continuum extrapolation and finite size scaling are needed. Spectroscopies around the pseudo-critical temperature were also performed. We confirmed wavefunctions are spatially localized, which implies hadrons 

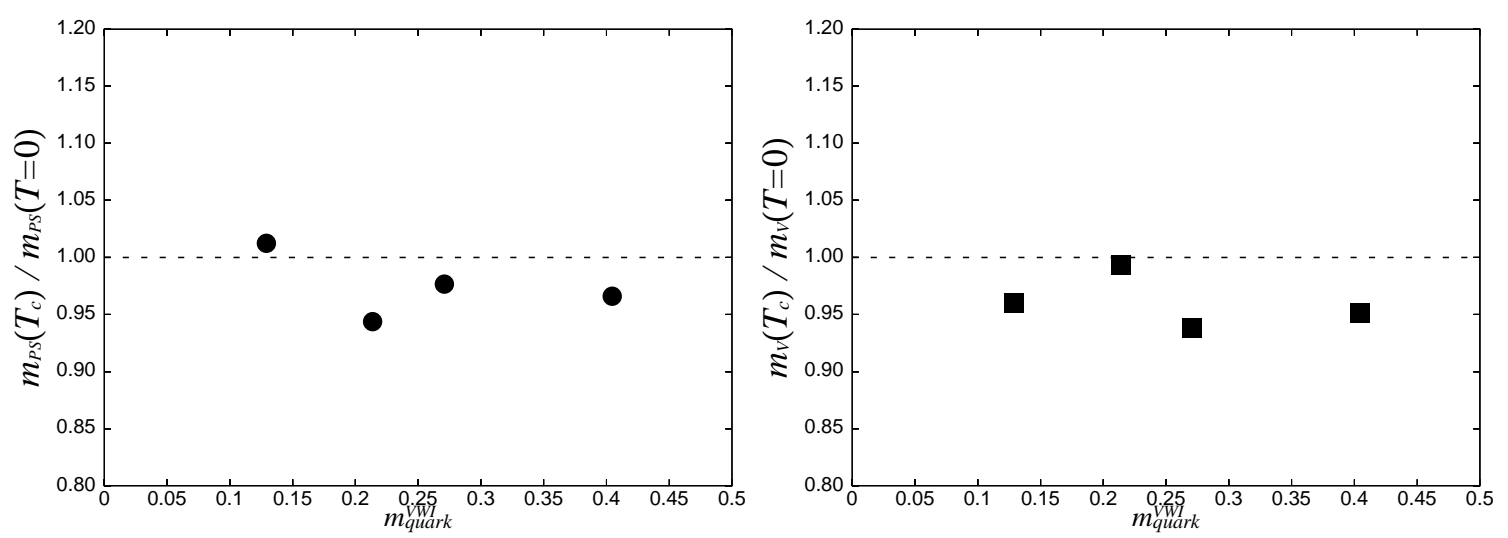

Figure 5: Sea quark mass dependence of pseudoscalar(left panel) and vector meson masses(right panel) at finite temperatures on $N_{t}=8$ lattices. Masses at zero temperature are used for normalization.

are bound states even near the pseudo-phase transition point. Using the measured wavefunctions for smearing, we extracted meson masses. On lattices with $N_{t}=8$, we found the pseudoscalar and vector meson masses do not show any significant shifts from their zero temperature values. Calculations on $N_{t}=10,12$ are ongoing toward the continuum extrapolation.

\section{Acknowledgments}

We thanks M. Harada and K. Kanaya for valuable discussions. This work is supported in part by $21^{\text {st }}$ Century COE Program, Nagoya University and by Grants-in-Aid of the Ministry of Education (No. 18740139 ). Numerical simulations were performed on clusters at Center for Computational Sciences, University of Tsukuba, and on Blue Gene at High Energy Accelerator Research Organization (KEK) under a support of the Large Scale Simulation Program.

\section{References}

[1] For a recent review, see

F. Karsch, J. Phys. Conf. Ser. 46 (2006) 122; U. M. Heller, PoS (LAT2 006 ) 011.

[2] Y. Aoki et al., Nature 443 (2006) 675.

[3] S. R. Sharpe, PoS (LAT2006) 022.

[4] CP-PACS Collaboration, A. Ali Khan et al., Phys. Rev. D 63 (2001) 034502, ibid. 64 (2001) 074510; Y. Maezawa et al., J.Phys.G 34 (2007) S651.

[5] V.G. Bornyakov et al., POS (LAT2005) 157.

[6] Y. Iwasaki, Nucl. Phys. B 258 (1985) 141; Univ. of Tsukuba report (1983) UTHEP-118, unpublished; S. Ejiri et al., Phys. Rev. D 68 (2003) 014502.

[7] CP-PACS Collaboration, T. Umeda et al., Phys. Rev. D 68 (2003) 034503.

[8] R.D. Pisarski and F. Wilczek, Phys. Rev. D 29 (1984) 338.

[9] K. Kanaya and S. Kaya, Phys. Rev. D 51 (1995) 2404.

[10] M. Harada et al., Phys. Lett. B 568 (2003) 103. 